\title{
SOBRE EL CARACTER SELECTIVO DE LAS PRUEBAS DE ACCESO A LA UNIVERSIDAD
}

Manuel Justel y Ubaldo Martínez-Lázaro

\section{Introducción}

Nadie niega hoy que la Universidad española es una institución clasista. Desde distintas perspectivas ideológicas, se afirma que la Universidad ocupa un lugar destacado, si no central, en la vertebración y desarrollo de una sociedad clasista, tanto por su papel en el reclutamiento y formación de sus cuadros directivos cuanto por su contribución a la elaboración y difusión del discurso ideológico legitimador de las desigualdades reales de la sociedad.

En sentido más restringido se habla del clasismo universitario en referencia a la composición del estudiantado y su extracción social. En este sentido, en las modernas sociedades occidentales el acceso a los estudios superiores de rango universitario parece constituir en buena medida un privilegio, si no de la «clase dominante», sí de los estratos medios y altos. En efecto, los hijos de los trabajadores manuales que acceden a la Universidad son una clara minoría. Aun en los Estados Unidos y Canadá, donde el paso por la Universidad está mucho más generalizado, los hijos de trabajadores manuales están considerablemente infrarrepresentados entre los universitarios. 
En España se asegura que se dan estos mismos supuestos y más acentuados si cabe que en la mayor parte de los países de la Europa Occidental '. El hecho de que la mayoría de los estudios existentes, referidos a los últimos años, coincidan en afirmar que apenas un 10 por 100 de los universitarios españoles son hijos de trabajadores manuales, cuando éstos representan aproximadamente el 40 por 100 de la población activa, pone al descubierto la existencia real del clasismo en la Universidad española ${ }^{2}$.

En estas páginas vamos a referirnos al "clasismo» que dice relación a los origenes sociales del estudiantado. Sobre todo al papel que las actuales pruebas de acceso a la Universidad juegan en el reclutamiento diferencial del alumnado, tratando de verificar en qué medida estas pruebas contribuyen a ese «clasismo» antes aludido.

Los datos utilizados para este estudio proceden básicamente de una de las encuestas más completas existentes hasta el momento sobre los estudiantes que se presentan a las pruebas de acceso a la Universidad; en concreto, los referidos a los estudiantes que pretendían el ingreso en la Universidad para el curso $1979-1980^{3}$.

Las pruebas de «selectividad» fueron presentadas en su momento como un intento de paliar la masificación de la Universidad. Desde el principio de la década de los sesenta el número de universitarios había comenzado un proceso de crecimiento excepcional. El mayor bienestar, derivado del des-

'C. Manuel SACRISTÁN, "La Universidad y la división del Trabajo", en Argumentos, n. 6 (1977), pp. 37-48 (publicado anteriormente en Realidad, n. 21 , septiembre de 1971); Félix OrTEGa, "Educación, movilidad social y selectividad", en Bordón, $\mathrm{n}^{\circ} 213$ (1976); J. Navarro, "Educación y desigualdad social", en Documentación Social, n. 23 (1976), y V. Bozal y L. Paramio, "Sistema educativo, sistema de clase", en Zona Abierta, n. 2 (1975).

2 Véanse, entre otros, los trabajos de M.' Angeles Durán Heras, "Procedencia social de los universitarios españoles", en Boletín del Centro de Estudios Sociales, n. ${ }^{1}$ (1968); "Universidad y lucha de clases", en Sistema, nn. 24-25 (1978), pp. 77-87, y "El origen social de los estudiantes universitarios", en su libro El trabajo de la mujer universitaria en la empresa, Fundación Universidad Empresa, Madrid, 1977. También, Salustiano DEL CAMpo, "Procedencia social del universitario", en Cambios sociales y formas de vida, Ariel, Barcelona, 1968; Cubells, F., "¿El origen social de los estudiantes condiciona su éxito académico?, en Revista de Ciencias de la Educación, n. ${ }^{\circ} 69$ (1972); LoREnzo GeLices, F., "Procedencia social de los universitarios", en Revista de Educación, n. ${ }^{\circ} 12$ (1963).

${ }^{3}$ Los datos principales contenidos en este trabajo proceden de un breve cues. tionario administrado a 74.238 de los 93.314 aspirantes al ingreso en la Universidad para el curso 1979-1980. De los 19.076 alumnos restantes, correspondientes a las Universidades de Navarra, Salamanca, Granada y Politécnica de Barcelona, en las que por distintas razones no pudo aplicarse el cuestionario, se conoce su distribución por regiones y provincias y la proporción de aprobados y suspensos. Como esta proporción coincide puntualmente con la del resto, creemos que cabe generalizar a nivel nacional los datos referidos a los 74.238 alumnos. Agradecemos al entonces Ministerio de Universidades e Investigación, así como a Rafael López Pintor, Miguel Beltrán y Margarita Gómez Vispo, que redactaron una primera presentación de los datos de la encuesta, el que hayan tenido la amabilidad de ponerlos a nuestra disposición. La codificación y el proceso de datos se ha realizado en el Centro de Investigaciones Sociológicas. 
arrollo económico, la generalización de altas expectativas educativas como clave del éxito futuro y el acceso generalizado de la mujer a los estudios universitarios fueron otros tantos factores que provocaron la multiplicación del número de estudiantes universitarios, hasta desbordar ampliamente los recursos existentes en la Universidad ${ }^{4}$. Esto, unido a una supuesta falta de preparación de los bachilleres y la preocupación por un alto porcentaje de fracasos en la fase de estudios universitarios, motivó presumiblemente la creación de dichas pruebas.

Su creación recibió una acogida hostil por parte de la opinión pública, especialmente viva entre los profesionales de clase media (de lo que quizá sea sintomática la intensa campaña de prensa dirigida contra ella) y la entonces clandestina oposición de izquierdas, muy singularmente en los medios universitarios.

Muchas fueron las críticas dirigidas contra la «selectividad». Fue acusada, probablemente con justicia, de tecnocrática y meritocrática; de olvidar las exigencias individuales y sociales de promoción cultural, de enriquecimiento individual y de bienestar colectivo; sobre todo, porque contemporáneamente no se hacía una política alternativa de asignación a la Universidad de mayores recursos y se sometía unc de los bastiones de la cultura a las exigencias del proceso productivo y el enriquecimiento intelectual a los estrechos criterios del éxito académico ${ }^{5}$.

Una acusación se repitió con especial insistencia y tuvo una acogida privilegiada entre los detractores de la ley: su carácter clasista. Se razonaba, en efecto, que la ley reforzaría el clasismo del sistema universitario, al restringir el acceso a la Universidad de los estudiantes de origen modesto. Estos, se afirmaba, gozan desde el nacimiento de menores oportunidades de acceso a la cultura. El haber sido educados inicialmente en ambientes menos ricos en estímulos culturales hace que salgan perjudicados al formar parte de un sistema educativo claramente jerarquizado y competitivo, en el que, además, son objeto de un trato desigual en cuanto a los medios y terminan por ser discriminados a la hora del resultado final. Esta discriminación selectiva tendría lugar, en buena medida, a la hora de intentar el acceso a la Universidad.

No se niegan aquí, en absoluto, los bandicaps y desigualdades de un sistema educativo claramente clasista. Por el contrario, se insiste en que las

4 A este respecto, véase la discusión del tema en Matilde VÁzquez, "¿Es la Universidad española una Universidad de masas?", en Documentación Social, n. ${ }^{\circ} 23$ (176), pp. 131-142.

s Sobre el tema de la selectividad, véanse los trabajos de N. ORTEGa, "Reflexiones en torno a la selectividad"; V. ORTEGA, "¿Nueva o vieja política de selectividad universitaria?", y S. SÁnchez Cerezo, "La selectividad, iinjerencia o parasistema?", publicados en el número monográfico de Documentación Social, n. ${ }^{\circ} 15$ (1974). Véase también la "Encuesta sobre la reforma universitaria" (Sistema, números 24-25, 1978, pp. 117-208), en la que participan una treintena de profesores universitarios de distintos planteamientos ideológicos y de diferentes ramas dentro del mundo académico. 
oportunidades de acceso a la educación y la cultura, tanto las escolares como las extraescolares, están claramente diferenciadas y discriminan a los alumnos de origen modesto.

De todos modos, el énfasis habría que situarlo más en los niveles previos a la enseñanza universitaria y menos en la fuerza selectiva de las pruebas de acceso a la Universidad y de la Universidad misma. En todo caso, la Universidad coronaría ese proceso selectivo, efectuado con anterioridad, al confirmarlo simbólicamente y al evidenciarlo en los resultados finales. Esta es nuestra hipótesis básica.

Es perfectamente posible, en el contexto de este razonamiento, que por este carácter clasista del sistema educativo los estudiantes de origen obrero que hayan logrado superar las trabas y obstáculos de un sistema hostil hacia ellos en los niveles previos a la Universidad sean aquéllos dotados de cualidades notables, si no excepcionales. Por esas aptitudes y una notable adaptación a un sistema que, en principio, les era hostil, cabría perfectamente pensar que su calificación en esas pruebas de acceso podría igualar, y hasta aventajar, en ciertos casos, la de sus compañeros de más alta extracción social.

En todo caso, cabe pensar que la socorrida afirmación del carácter marcadamente clasista de la «selectividad», es decir, de las pruebas actuales de acceso a la Universidad, no es ni mucho menos evidente y que ha de ser contrastada de forma rigurosa con los datos disponibles. Sólo así será posible medir su alcance selectivo y su fuerza socialmente discriminadora ${ }^{6}$.

\section{Clasismo del sistema educativo español}

El carácter clasista del sistema educativo español es fácilmente perceptible si se analizan algunos datos relativos a la proporción de estudiantes que finalizan cada etapa educativa comparando su distribución a partir de algunas variables con las proporciones respectivas de población o proporción esperada de estudiantes. En líneas generales, la proporción esperada de estudiantes en cada etapa educativa de un sistema no clasista habría de coincidir con la proporción de población en cada categoría de la variable. Evidentemente, esto no es así en el caso español.

El cuadro 1 recoge datos del censo de 1970 . Se refiere a la categoría socioprofesional del padre y evidencia el marcado carácter clasista del siste-

- Una aportación interesante en este sentido puede verse en el artículo ya citado de Félix ORTEGa sobre Educación, movilidad social y selectividad. La educación y el conflicto de clases (en Bordón, $\mathfrak{n} .^{\circ} 213,1976$, pp. 181-202). En él critica las concepciones psicologistas, espiritualistas y funcionalistas de la educación y pone especial énfasis en la base clasista de cualquier sistema selectivo de enseñanza, mediante el análisis de algunas variables socio-económicas y ambientales. 


\section{CUADRO 1}

Acceso a los distintos niveles educativos según la categoría socio-profesional del padre (1970)

\begin{tabular}{|c|c|c|c|c|c|c|c|c|c|}
\hline \multirow{2}{*}{$\begin{array}{l}\begin{array}{l}\text { Categoria socio-profesional } \\
\text { del padre }\end{array} \\
\begin{array}{l}\text { Cuadros superiores y pro- } \\
\text { fesiones liberales .. ... }\end{array}\end{array}$} & \multicolumn{2}{|c|}{$\begin{array}{l}\text { de po- } \\
\text { blación } \\
\text { activa } \\
\text { masculina }\end{array}$} & \multicolumn{2}{|c|}{$\begin{array}{l}\text { Bachille- } \\
\text { rato ele- } \\
\text { mental } \\
(\%)\end{array}$} & \multicolumn{2}{|c|}{$\begin{array}{l}\text { Bachille- } \\
\text { rato supe- } \\
\text { rior (or) }\end{array}$} & \multicolumn{2}{|c|}{$\begin{array}{l}\text { Univer- } \\
\text { sidad }\end{array}$} & $\begin{array}{l}\text { Estu- } \\
\text { diantes } \\
\text { universi- } \\
\text { tarios por } \\
\text { cada } \\
10.000 \\
\text { personas } \\
\text { activas }\end{array}$ \\
\hline & 3,37 & & 7,97 & & 14,9 & & 31,97 & & 1.633 \\
\hline Cuadros medios & 4,1 & & 4.4 & & 11.9 & & 14.6 & & 599 \\
\hline $\begin{array}{l}\text { Empresarios con asalaria- } \\
\text { dos de la industria y los } \\
\text { servicios } \ldots \begin{array}{lllll} & \ldots & \ldots & \ldots & \ldots\end{array}\end{array}$ & 3,1 & 12,0 & 6.0 & 20.0 & 7.5 & 37,1 & 7,6 & 57.2 & 419 \\
\hline \begin{tabular}{llllll} 
Empresarios & \multicolumn{2}{c}{ agrarios } & \multicolumn{1}{c}{ con } \\
asalariados & $\ldots$ & $\ldots$ & $\ldots$ & $\ldots$ & $\ldots$ \\
Euerzas $A r m a d a s$ & $\ldots$ & $\ldots$ & $\ldots$ \\
Empleados & $\ldots$ & $\ldots$ & $\ldots$ & $\ldots$ & $\ldots$
\end{tabular} & $\begin{array}{r}1,5 \\
1,5 \\
10.3\end{array}$ & & $\begin{array}{r}1,9 \\
3,8 \\
14,9\end{array}$ & & $\begin{array}{r}2,8 \\
5,4 \\
17,8\end{array}$ & & $\begin{array}{r}3,1 \\
5,1 \\
15,3\end{array}$ & & $\begin{array}{l}342 \\
563 \\
251\end{array}$ \\
\hline $\begin{array}{l}\text { Empresarios sin asalaria- } \\
\text { dos y trabajadores inde- } \\
\text { pendientes } \ldots \ldots \ldots\end{array}$ & 6,9 & 23.4 & 8,9 & 32,9 & 8. & 35.6 & 6 , & 29.2 & 146 \\
\hline Personal de servicios $\ldots . .$. & $4,7]$ & & 5,3 & & $\left.\begin{array}{l}0,0 \\
4,1\end{array}\right]$ & & 2,8 & & 100 \\
\hline Obreros calificados .... & 30.7 & & 27,5 & & 15,27 & & 7,07 & & 38 \\
\hline $\begin{array}{c}\text { Personal calificado en la } \\
\text { agricultura } \ldots \ldots \\
.\end{array}$ & 2,6 & & 1,5 & & 1,2 & & 0,7 & & $\begin{array}{l}47 \\
51\end{array}$ \\
\hline Agricultores $\sin$ asalariados & 13,1 & 64,5 & 7,1 & 43.9 & 6,8 & 27.3 & 4.0 & 13,5 & 51 \\
\hline $\begin{array}{llll}\text { Obreros } \sin \text { calificar } & \ldots & \ldots \\
\text { Jornaleros del campo } & . . & \ldots\end{array}$ & $\begin{array}{r}8,1 \\
10,0\end{array}$ & & $\left.\begin{array}{l}4,4 \\
3,4\end{array}\right]$ & & $\begin{array}{l}2,1 \\
2,0\end{array}$ & & $\begin{array}{l}1,0 \\
0,8\end{array}$ & & $\begin{array}{l}21 \\
14\end{array}$ \\
\hline 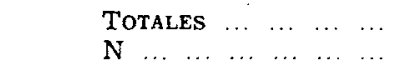 & $\begin{array}{l}100,0 \\
(9.285\end{array}$ & & $\begin{array}{l}100,0 \\
(1.236 .\end{array}$ & $850)$ & $\begin{array}{l}100,0 \\
(329\end{array}$ & $292)$ & $(156.7$ & $\begin{array}{l}100,0 \\
762)\end{array}$ & \\
\hline
\end{tabular}

Fuente: I. N. E., Censo de la Población de España, 1970, vol. III. Se han excluido de las bases los retirados, rentistas y ocupaciones no clasificables, asi como las personas económicamente no activas.

$\mathrm{ma}^{7}$. Casi un tercio de los alumnos de la Universidad española son hijos de profesionales liberales y cuadros superiores, mientras que éstos no constituyen más que el 3,3 por 100 de la población activa masculina. Por el contra-

Se utilizan aquí estos datos sin entrar en discusión con los criterios de agrupación profesional seguidos. Que los datos se refieran a 1970 no resta interés ni fuerza argumental a su contenido respecto del "clasismo" del sistema educativo en España. Cf. FOESSA, "Educación y Ciencia", en Informe sociológico sobre la situación social de España, 1970, Madrid, Euramérica, cap. 14, y FOESSA, "Educación", en Estudios sociológicos sobre la situación socíal de España, Madrid, Euramérica, cap. 2. Véase también José Navarro, "Educación y desigualdad social", en Documentación Social, n. 23 (1976), pp, 49-63. 
rio, los obreros calificados son casi un tercio de la población activa masculina y sus hijos presentes en la Universidad representan aproximadamente el 7 por 100 del total. Es decir, los primeros tienen una proporción de alumnos universitarios diez veces superior a su proporción de población y los segundos

\section{CUADRO 2}

Aspirantes a la Universidad por profesión del padre

(Proporción de aspirantes al ingreso en la Universidad, 1979-1980, según categoría socio-profesional del padre y según porcentaje de población activa masculina que representa)

\begin{tabular}{|c|c|c|c|}
\hline Profesión del padre & TOTAL & $\begin{array}{l}\text { "pobla- } \\
\text { ción } \\
\text { activa }\end{array}$ & $\begin{array}{l}\text { \%raspi- } \\
\text { rantes }\end{array}$ \\
\hline Empresarios agrarios con asalariados $\ldots \ldots \ldots \ldots$ & (941) & 1,4 & 1,27 \\
\hline $\begin{array}{l}\text { Empresarios agrarios sin asalariados y miembros } \\
\text { de cooperativas de producción agraria } \ldots \ldots \ldots\end{array}$ & $(4.500)$ & 8,7 & 6,05 \\
\hline $\begin{array}{llllllllll}\text { Trabajadores agrarios } & \ldots & \ldots & \ldots & \ldots & \ldots & \ldots & \ldots & \ldots & \ldots\end{array}$ & (1.376) & 10,8 & 1,86 \\
\hline Empresarios no agrarios con asalariados $\ldots \ldots \ldots$ & $(10.863)$ & 3,8 & 14,63 \\
\hline $\begin{array}{c}\text { Empresarios no agrarios } \sin \text { asalariados y traba- } \\
\text { jadores independientes } \ldots \ldots \\
\ldots\end{array}$ & $(3.224)$ & 6,8 & 4,34 \\
\hline $\begin{array}{l}\text { Profesionales liberales y asimilados de activida- } \\
\text { des por cuenta propia con o sin asalariados ... }\end{array}$ & $(3.465)$ & 1,5 & 4,67 \\
\hline $\begin{array}{c}\text { Directores y gerentes de empresas y sociedades } \\
\text { no agrarias } \\
\ldots\end{array}$ & $(3.268)$ & 2,0 & 4,40 \\
\hline 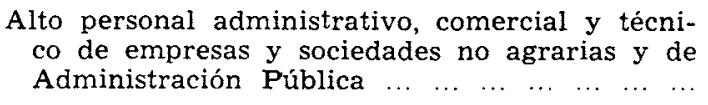 & $(6.153)$ & 1,9 & 8.30 \\
\hline $\begin{array}{l}\text { Personal intermedio administrativo, comercial y } \\
\text { técnico de empresas y sociedades no agrarias } \\
\text { y de Administración Pública ... ... . . . . . . . . }\end{array}$ & (4.544) & 4,9 & 6,12 \\
\hline $\begin{array}{l}\text { Resto personal administrativo, comercial y téc- } \\
\text { nico de empresas y sociedades no agrarias y } \\
\text { de Administración Pública } \ldots \ldots \ldots c c c c c c \\
\text { Ad }\end{array}$ & $(10.924)$ & 9,3 & 14,71 \\
\hline $\begin{array}{l}\text { Contramaestres, capataces y asimilados no agra- } \\
\text { rios, jefes de grupos y otros trabajadores de } \\
\text { servicios } \ldots \ldots \ldots \\
\end{array}$ & $(2.497)$ & 5,7 & 3,36 \\
\hline Obreros calificados y especializados no agrarios. & $(12.948)$ & 24,7 & 17,44 \\
\hline Obreros sin especialización no agrarios $\ldots \ldots \ldots$ & $(2.208)$ & 7.0 & 2,97 \\
\hline Profesionales de las Fuerzas Armadas ... ... ... & $(3.703)$ & 2,2 & 4,99 \\
\hline 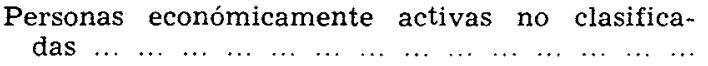 & $(3.624)$ & 1,7 & 4,89 \\
\hline
\end{tabular}


una proporción cinco veces menor a la de su población activa masculina. En la última columna del cuadro se aprecian aún mejor esas desproporciones. En los niveles educativos anteriores a la Universidad también existen desproporciones importantes, como puede apreciarse en el cuadro mencionado. El sesgo clasista del sistema educativo aparece con nitidez en el cuadro 2, que muestra la distribución, según la ocupación paterna, de los estudiantes presentados a las pruebas de acceso a la Universidad en 1979.

Casi un tercio de los aspirantes (un 32 por 100 exactamente) son hijos de empleados de la industria y los servicios, cuadros superiores de la Administración y la empresa privada o profesionales liberales que, en conjunto, suponen menos de un 10 por 100 de la población activa. Por el contrario, poco más de una cuarta parte de los estudiantes son hijos de trabajadores manuales que suponen, en números redondos, la mitad de la población activa.

El cuadro mencionado es doblemente interesante en cuanto recuerda la complejidad del sistema de desigualdades que caracterizan nuestra estructura social. Las tres primeras categorías de dicho cuadro muestran un sector agrario que, aunque claramente jerarquizado, está en neta desventaja colectiva en cuanto a las oportunidadcs de acceso a la educación. Los hijos de los trabajadores agrícolas (más de un 10 por 100 de la población activa) suponen menos de un 2 por 100 de los candidatos. Incluso los empresarios con asalariados están ligeramente infrarrepresentados entre los futuros universitarios.

Las desigualdades en base a la profesión se articulan y refuerzan con las ecológico-estructurales, en concreto con la dimensión rural-urbana. Incluso en la distribución por provincias del alumnado universitario de 1970 se aprecia esto mismo y se sigue apreciando actualmente en base al número de alumnos por provincia y región que se presentan a las pruebas de acceso para el curso 1979-1980 (ver los cuadros A y B del Anexo).

Atendiendo a la distribución por tamaño de hábitat de los alumnos presentados a las pruebas de acceso a la Universidad, se aprecia claramente la sobrerrepresentación urbana que existe. Así queda reflejado en el cuadro 3, en el que se compara la proporción de alumnos candidatos a la Universidad con la proporción de población para cada estrato o tamaño de hábitat.

Destaca, por su menor densidad de alumnado, el estrato de 2.000 a 10.000 habitantes, con una desproporción de 2 a 1 entre población y alumnos. El envejecimiento actual de la población rural española juega en ello un papel que no hay que olvidar, aunque no explique por sí solo esta gran desproporción. Por su mayor densidad de alumnado destaca el estrato poblacional de más de un millón de habitantes: su proporción de alumnos es una cuarta parte superior a la de su población.

En general, los datos evidencian una sobrerrepresentación urbana y una infrarrepresentación rural. Al 50,7 por 100 de la población que, según el censo de 1970, habita en municipios de menos de 50.000 habitantes le corres- 
ponde el 35 por 100 de los estudiantes que intentan ingresar en la Universidad; por el contrario, al 49,3 por 100 de la población que habita en municipios de más de 50.000 habitantes (población claramente urbana) le corresponde el 65 por 100 del total de los alumnos.

\section{CUADRO 3}

Distribución por tamaño de hábitat de los alumnos presentados a las pruebas de acceso a la Universidad (1979-1980)

\begin{tabular}{|c|c|c|}
\hline Tamaño de hábitat & $\underset{(\%)}{\text { Alumnos }}$ & $\begin{array}{c}\text { Población } \\
(\%)\end{array}$ \\
\hline Menos de 2.000 habitantes $\ldots \ldots \ldots c c c c$ & 6.6 & 11.2 \\
\hline De $\quad 2.001$ a $\quad 10.000$ habitantes $\ldots \ldots \ldots \ldots$ & 11.1 & 21.5 \\
\hline 50.000 habitantes $\ldots \ldots \ldots \ldots$ & 17.3 & 18.0 \\
\hline De $\quad 50.001$ a 100.000 habitantes $\ldots \ldots \ldots \ldots$ & 8.5 & 5.0 \\
\hline De 100.001 a 400.000 habitantes $\ldots \ldots \ldots \ldots$ & 17.8 & 16.2 \\
\hline De 400.001 a 1.000 .000 de habitantes $\ldots \ldots \ldots$ & 11.4 & 8.7 \\
\hline Más de 1.000 .000 de habitantes $\ldots \begin{array}{llll}\ldots & \ldots & \ldots & \ldots\end{array}$ & 26.0 & 19.4 \\
\hline $\begin{array}{llllllllllll}\text { No } p \text { rocede } & \ldots & \ldots & \ldots & \ldots & \ldots & \ldots & \ldots & \ldots & \ldots & \ldots & \ldots\end{array}$ & 1.3 & - \\
\hline $\begin{array}{lllllllll}\text { TOTAL } & \ldots & \ldots & \ldots & \ldots & \ldots & \ldots & \ldots & \ldots\end{array}$ & 100.00 & 100.00 \\
\hline
\end{tabular}

* Censo de 1970.

Se puede afirmar, por tanto, que el medio rural o cuasi-rural está estructuralmente discriminado en cuanto a oportunidades de acceso a la Universidad. Los alumnos procedentes del medio rural llegan a presentarse a las pruebas de acceso a la Universidad en proporción notablemente inferior a los alumnos procedentes del medio urbano.

Fuerza «selectiva» de las pruebas de acceso a la Universidad

Las diferencias relativas de éxito que presentan los alumnos de origen modesto ante las pruebas de acceso explican sólo en muy pequeña medida la gran desproporción de los mismos que se da, de hecho, entre el alumnado universitario.

De todos modos existe un mayor índice de fracasos entre los alumnos de procedencia rural: los aprobados son proporcionalmente más cuanto mayor es el volumen de la población de los municipios de origen de los estudiantes 
que se examinan y menos en la medida que su municipio de origen es menor. Los aprobados del estrato intermedio (de 50.000 a 100.000 habitantes) coinciden en proporción con el promedio nacional. La proporción es apreciablemente inferior (y decrece) en los estratos inferiores, alcanzando el mínimo en los municipios de menos de 2.000 habitantes. En estos últimos el porcentaje de aprobados es cinco puntos inferior al nacional. La proporción más alta (cinco puntos superior a la nacional) la ostentan los municipios entre 400.001 y 1.000 .000 de habitantes. Sin embargo, Madrid y Barcelona presentan un porcentaje dos puntos superior al nacional (ver cuadro 4 ).

\section{CUADRO 4}

Proporción de alumnos aprobados en las prucbas de acceso a la Universidad (1979-1980) por tamaño de bábitat

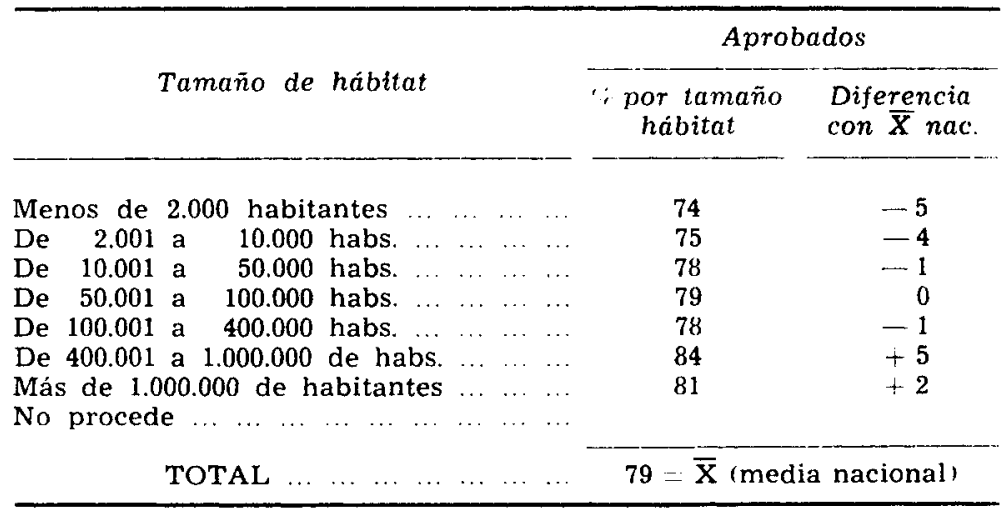

De acuerdo con el cuadro 5, el mayor porcentaje de aprobados corresponde a los hijos de los profesionales liberales y de los directivos y empleados de la industria privada ( 83 por 100). Los hijos de los trabajadores manuales de la industria y los servicios aprueban en proporción casi igual ( 78 por 100) a la media de la muestra ( 79 por 100). Este porcentaje de aprobados es superior al correspondiente a los hijos de profesores y maestros (78 por 100) y de empresarios de la industria y los servicios. Los hijos de los trabajadore: y jornaleros agrícolas, aunque representan una pequeña proporción de los que acceden a la prueba, aprueban en porcentaje dos puntos inferior al promedio. Hay que señalar que la categoría más castigada por la selectividad son los empresarios agrícolas, con un promedio de éxitos (72 por 100) muy inferior al correspondiente a los trabajadores manuales.

En relación a los empresarios (por su heterogeneidad) y trabajadores manuales, a los que se consideraba los principales perjudicados, hemos creído clarificador una consideración más detallada, como la resumida en el cuadro 6. 


\section{CUADRO 5}

Presentados y aprobados por profesión del padre

(Distribución de alumnos presentados y porcentaje de aprobados en las pruebas de acceso a la Universidad, 1979-1980, según categoría socio-profesional del padre)

\begin{tabular}{|c|c|c|c|c|}
\hline \multirow{2}{*}{$\begin{array}{l}\text { Categoria socio-profesional } \\
\text { del padre }\end{array}$} & \multicolumn{2}{|c|}{ Alumnos presentados } & \multirow{2}{*}{$\begin{array}{c}\% \text { de } \\
\text { aprobados }\end{array}$} & \multirow{2}{*}{$\begin{array}{l}\text { Diferencia } \\
\quad \text { con } \bar{X}\end{array}$} \\
\hline & Número & $\%$ & & \\
\hline $\begin{array}{l}\text { Propietarios y empresarios en agricul- } \\
\text { tura y silvicultura } \ldots \ldots \\
\end{array}$ & 5.465 & 7,4 & 72 & -7 \\
\hline $\begin{array}{l}\text { Obreros y trabajadores en agricultura } \\
\text { y silvicultura } \\
\ldots\end{array}$ & 1.389 & 1,9 & 77 & -2 \\
\hline $\begin{array}{l}\text { Empresarios en industria, comercio, } \\
\text { mineria, pesca, construcción, trans- } \\
\text { portes y servicios } \ldots \ldots \ldots \ldots \\
\end{array}$ & 13.943 & 18,8 & 77 & -2 \\
\hline $\begin{array}{l}\text { Obreros y trabajadores en industria, } \\
\text { comercio, minería, pesca, construc- } \\
\text { ción, transportes y servicios ... } \ldots\end{array}$ & 16.177 & 21,8 & 78 & -1 \\
\hline $\begin{array}{l}\text { Directores, profesionales } y \text { personal } \\
\text { empleado y de oficina en empresas } \\
\text { y sociedades privadas } \ldots \ldots \\
\end{array}$ & 16.184 & 21,8 & 83 & +4 \\
\hline $\begin{array}{c}\text { Funcionarios y empleados de la Admi- } \\
\text { nistración Pública } \\
\text {. }\end{array}$ & 5.935 & 8,0 & 81 & +2 \\
\hline $\begin{array}{lllllllll}\text { Fuerzas Armadas } & \ldots & \ldots & \ldots & \ldots & \ldots & \ldots & \ldots\end{array}$ & 3.709 & 5,0 & 78 & -1 \\
\hline $\begin{array}{lllllll}\text { Profesiones liberales } & \ldots & \ldots & \ldots & \ldots & \ldots\end{array}$ & 3.099 & 4,2 & 83 & +4 \\
\hline Profesiones de la docencia $\ldots \ldots \ldots \ldots$ & 5.221 & 7,0 & 77 & -2 \\
\hline Otras actividades profesionales $\ldots \ldots$ & 3.116 & 4,2 & 81 & +2 \\
\hline $\begin{array}{llllllll}\text { TOTAL } & \ldots & \ldots & \ldots & \ldots & \ldots & \ldots & \ldots\end{array}$ & 74.238 & 100,0 & - & - \\
\hline
\end{tabular}

Los hijos de los grandes empresarios industriales y los empresarios agrícolas grandes y medianos superan las pruebas en proporción considerablemente inferior a la correspondiente a cualquier categoría de trabajadores manuales. Con la excepción de los asalariados agrícolas fijos y los peones de la industria y los servicios, los hijos de trabajadores manuales aprueban en proporción similar a la media y, en algún caso, netamente superior. Hay que señalar, por significativo, el caso de los jornaleros agrícolas, cuyos hijos, aunque constituyen una insignificante minoría, superan la selectividad más airosamente que el promedio. 


\section{CUADRO 6}

\section{Aprobados por profesión del padre}

(Porcentaje de alumnos aprobados en las pruebas de acceso a la Universidad, 1979-1980, según algunas categorías socio-profesionales del padre)

\begin{tabular}{|c|c|c|c|}
\hline Categoria socio-profesional del padre & $\begin{array}{l}N \text { alumnos } \\
\text { presentados }\end{array}$ & $\begin{array}{l}\text { \% de } \\
\text { aprobados }\end{array}$ & $\begin{array}{l}\text { Diferencia } \\
\quad \operatorname{con} \bar{X}\end{array}$ \\
\hline $\begin{array}{llllll}\text { Grandes propietarios agrícolas } & \ldots & \ldots & \ldots & \ldots\end{array}$ & 30 & 70 & -9 \\
\hline Propietarios agricolas con asalariados fijos. & 243 & 70 & -9 \\
\hline 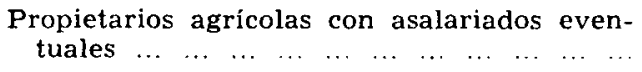 & 668 & 73 & -6 \\
\hline Propietarios agrícolas $\sin$ asalariados $\ldots \ldots$ & 4.500 & 72 & -7 \\
\hline $\begin{array}{lllllllll}\text { Grandes empresarios } & \ldots & \ldots & \ldots & \ldots & \ldots & \ldots & \ldots\end{array}$ & 162 & 69 & -10 \\
\hline $\begin{array}{lllllllll}\text { Empresarios } & \text { medios } & \ldots & \ldots & \ldots & \ldots & \ldots & \ldots & \ldots\end{array}$ & 4.148 & 78 & -1 \\
\hline $\begin{array}{llllllll}\text { Pequeños empresarios } & \ldots & \ldots & \ldots & \ldots & \ldots & \ldots & \ldots\end{array}$ & 654 & 78 & -1 \\
\hline $\begin{array}{c}\text { Empresarios sin asalariados, trabajadores in- } \\
\text { dependientes y artesanos } \\
\ldots\end{array}$ & 3.080 & 77 & -2 \\
\hline Trabajadores agrícolas especializados $\ldots \ldots$ & 101 & 85 & +6 \\
\hline $\begin{array}{llllllll}\text { Obreros agrícolas fijos } & \ldots & \ldots & \ldots & \ldots & \ldots & \ldots & \ldots\end{array}$ & 1.103 & 76 & -3 \\
\hline $\begin{array}{llllllllllll}\text { Jornaleros } & \ldots & \ldots & \ldots & \ldots & \ldots & \ldots & \ldots & \ldots & \ldots & \ldots & \ldots\end{array}$ & 172 & 80 & +1 \\
\hline Capataces de la industria y servicios ... & 2.497 & 79 & 0 \\
\hline $\begin{array}{llllllll}\text { Obreros especializados } & \ldots & \ldots & \ldots & \ldots & \ldots & \ldots & \ldots\end{array}$ & 11.925 & 78 & -1 \\
\hline $\begin{array}{lllllllllllll}\text { Peones } & \ldots & \ldots & \ldots & \ldots & \ldots & \ldots & \ldots & \ldots & \ldots & \ldots & \ldots & \ldots\end{array}$ & 1.737 & 76 & -3 \\
\hline
\end{tabular}

El clima cultural de la familia y, más en concreto, el nivel de estudios de los padres marca diferencias claras y unívocas respecto del éxito de los hijos a la hora de entrar en la Universidad. El mayor porcentaje de aprobados entre los hijos de directivos, empleados y profesionales se debe, sobre todo, al nivel cultural que suelen tener los titulares de esas profesiones.

En general, existe una asociación positiva entre el nivel de estudios del padre y la tasa de aprobados de los hijos (cuadro 7). Otro tanto se aprecia si se toma en consideración el nivel de estudios de la madre (cuadro 8). Y, como era de esperar, sucede lo mismo cuando se consideran conjuntamente los niveles culturales de ambos (cuadro 9). Con la particularidad de que el índice de aprobados de los hijos es exactamente el mismo, sea el padre o sea la madre quien tiene estudios superiores. Por supuesto, el índice de aprobados es más alto cuando ambos tienen estudios superiores; es ligeramente más bajo, pero todavía superior al medio, cuando cualquiera de ellos tiene estudios superiores, $y$ es inferior al índice medio cuando ninguno tiene estudios superiores. Esto indica claramente que, aunque el número de estudiantes universitarios está aumentando notablemente en todas las capas socia- 


\section{CUADRO 7}

Presentados y aprobados por nivel de estudios del padre (Distribución de alumnos presentados y porcentaje de aprobados en las pruebas de acceso a la Universidad, 1979-1980, según el nivel de estudios del padre)

\begin{tabular}{|c|c|c|c|c|}
\hline \multirow{2}{*}{ Nivel de estudios } & \multicolumn{2}{|c|}{ Alumnos presentados } & \multirow{2}{*}{$\begin{array}{c}\% \text { de } \\
\text { aprobados }\end{array}$} & \multirow{2}{*}{$\begin{array}{l}\text { Diferencia } \\
\quad \text { con } \bar{X}\end{array}$} \\
\hline & Número & $\%$ & & \\
\hline Titulación superior & 10.164 & 13,7 & 84 & +5 \\
\hline Título de grado medio $\ldots \ldots \ldots c c c c$ & 6.239 & 8,4 & 82 & +3 \\
\hline Bachillerato superior $\begin{array}{llllll}\ldots & \ldots & \ldots & \ldots & \ldots\end{array}$ & 8.432 & 11,4 & 81 & +2 \\
\hline $\begin{array}{lllllll}\text { Estudios profesionales } & \ldots & \ldots & \ldots & \ldots & \ldots\end{array}$ & 3.648 & 4,9 & 82 & +3 \\
\hline Bachiller elemental, E.G.B., primaria & 9.695 & 26,5 & 79 & 0 \\
\hline $\begin{array}{lllllllll}\operatorname{Sin} \text { estudios } & \ldots & \ldots & \ldots & \ldots & \ldots & \ldots & \ldots & \ldots\end{array}$ & 23.903 & 32,2 & 75 & -4 \\
\hline 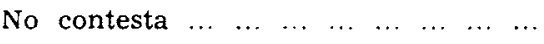 & 2.157 & 2,9 & - & - \\
\hline $\begin{array}{llllllll}\text { TOTAL } & \ldots & \ldots & \ldots & \ldots & \ldots & \ldots & \ldots\end{array}$ & 74.238 & 100,0 & - & - \\
\hline
\end{tabular}

les, el índice de reproducción de la capa social con alguna persona en la familia de nivel universitario es más alto, lo que mantiene y refuerza su carácter elitista.

\section{CUADRO 8}

Presentados y aprobados por nivel de estudios de la madre

(Distribuciór de alumnos presentados y porcentaje de aprobados en las pruebas de acceso a la Universidad, 1979-1980, según el nivel de estudios de la madre)

\begin{tabular}{|c|c|c|c|c|}
\hline \multirow{2}{*}{ Nivel de estudios } & \multicolumn{2}{|c|}{ Alumnos presentados } & \multirow{2}{*}{$\begin{array}{c}\% \text { de } \\
\text { aprobados }\end{array}$} & \multirow{2}{*}{$\begin{array}{l}\text { Diferencia } \\
\quad \text { con } \bar{X}\end{array}$} \\
\hline & Número & $\%$ & & \\
\hline 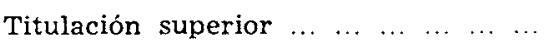 & 1.637 & 2,2 & 88 & +9 \\
\hline Título de grado medio $\ldots \ldots \ldots \ldots \ldots$ & 4.066 & 5,5 & 84 & +5 \\
\hline Bachillerato superior $\quad \ldots \begin{array}{llllll} & \ldots & \ldots & \ldots & \ldots & \ldots\end{array}$ & 7.741 & 10,4 & 82 & +3 \\
\hline Estudios profesionales $\ldots \ldots \ldots c c c c$ & 1.107 & 1,5 & 85 & +6 \\
\hline Bachiller elemental, E.G.B., primaria & 25.405 & 34,2 & 81 & +2 \\
\hline $\begin{array}{llllllllll} & \operatorname{Sin} \text { estudios } & \ldots & \ldots & \ldots & \ldots & \ldots & \ldots & \ldots & \ldots\end{array}$ & 31.180 & 42,0 & 76 & -3 \\
\hline $\begin{array}{lllllllll}\text { No contesta } & \ldots & \ldots & \ldots & \ldots & \ldots & \ldots & \ldots & \ldots\end{array}$ & 3.102 & 4,2 & - & - \\
\hline $\begin{array}{lllllllll}\text { TOTAL } & \ldots & \ldots & \ldots & \ldots & \ldots & \ldots & \ldots\end{array}$ & 74.238 & 100,0 & - & - \\
\hline
\end{tabular}




\section{CUADRO 9}

Presentados y aprobados por nivel de estudios de los padres (Distribución de alumnos presentados y porcentaje de aprobados en las pruebas de acceso a la Universidad, 1979-1980, según el nivel de estudios de los padres)

\begin{tabular}{|c|c|c|c|c|c|}
\hline \multirow{2}{*}{\multicolumn{2}{|c|}{ Nivel de estudios }} & \multicolumn{2}{|c|}{ Alumnos presentados } & \multirow{2}{*}{$\begin{array}{c}\% \text { de } \\
\text { aprobados }\end{array}$} & \multirow{2}{*}{$\begin{array}{l}\text { Diferencia } \\
\quad \operatorname{con} \bar{X}\end{array}$} \\
\hline & & Número & $\%$ & & \\
\hline Estudios & superiores ambos $\ldots \ldots \ldots$ & 3.917 & 5,3 & 86 & +7 \\
\hline Estudios & superiores padre $\ldots \ldots \ldots$ & 19.648 & 17,0 & 83 & +4 \\
\hline Estudios & superiores madre $\ldots \ldots \ldots \ldots$ & 1.930 & 2,6 & 83 & +4 \\
\hline \multirow[t]{2}{*}{ Ninguno } & estudios superiores $\ldots \ldots$... & 55.743 & 75,1 & 77 & -2 \\
\hline & $\begin{array}{llllllll}\text { TOTAL } & \ldots & \ldots & \ldots & \ldots & \ldots & \ldots & \ldots\end{array}$ & 74.238 & 100,0 & - & - \\
\hline
\end{tabular}

Los alumnos que intentan el acceso a la Universidad proceden mayoritariamente de centros estatales de enseñanza ( 52 por 100) (cuadro 10). Los centros religiosos preparan al 33 por 100 y los centros privados no religiosos un 13 por 100. Estos últimos coinciden en aprobados con el promedio; sin embargo, existe una diferencia notable entre centros estatales y centros religiosos. Estos consiguen un porcentaje de aprobados ocho puntos superior al de los estatales y cinco puntos superior al medio. El porcentaje más bajo lo consiguen los estatales con tres puntos inferior a la media.

\section{CUADRO 10}

Presentados y aprobados según tipo de centro

(Distribución de alumnos presentados y porcentaje de aprobados en las pruebas de acceso a la Universidad, 1979-1980, según el tipo de centro en que realizaron sus estudios)

\begin{tabular}{|c|c|c|c|c|c|}
\hline & \multirow{2}{*}{ Clase de centro } & \multicolumn{2}{|c|}{ Alumnos presentados } & \multirow{2}{*}{$\begin{array}{c}\% \text { de } \\
\text { aprobados }\end{array}$} & \multirow{2}{*}{$\begin{array}{l}\text { Diferencia } \\
\quad \operatorname{con} \overline{\bar{X}}\end{array}$} \\
\hline & & Número & $\%$ & & \\
\hline Estatal & $\begin{array}{lllllllll}\ldots & \ldots & \ldots & \ldots & \ldots & \ldots & \ldots & \ldots & \ldots\end{array}$ & 38.614 & 52 & 76 & -3 \\
\hline Religioso & $\begin{array}{lllllllll}\ldots & \ldots & \ldots & \ldots & \ldots & \ldots & \ldots & \ldots & \ldots\end{array}$ & 24.482 & 33 & 84 & +5 \\
\hline Seglar ... & $\begin{array}{lllllllll}\ldots & \ldots & \ldots & \ldots & \ldots & \ldots & \ldots & \ldots & \ldots\end{array}$ & 9.702 & 13 & 79 & 0 \\
\hline Varios $\ldots$ & $\begin{array}{lllllllll}\ldots & \ldots & \ldots & \ldots & \ldots & \ldots & \ldots & \ldots & \ldots\end{array}$ & 613 & 2 & 62 & -17 \\
\hline
\end{tabular}


Dado que a los centros privados suelen acudir en mayor número los hijos de familias acomodadas o ricas - puesto que no son gratuitos o lo son sólo parcialmente- aparece aquí un factor de discriminación en base al status socioeconómico de la familia a la hora del acceso de los hijos a la Universidad. Esta discriminación se produce en la medida en que el acceso está directamente relacionado con el tipo de centro en que el alumno ha realizado los estudios, siendo especialmente eficaces los centros privados y, entre ellos, los dirigidos por instituciones religiosas; y son precisamente estos centros en los que no pueden cursar sus estudios con igualdad de oportunidades los hijos de familias modestas (especialmente numerosas, entre la clase obrera), dado su actual coste económico.

\section{CUADRO 11}

Presentados y aprobados por clase social y expediente

(Distribución de alumnos presentados a las pruebas de acceso a la Universidad, 1979-1980, según clase social del padre y según la nota media del expediente académico)

\begin{tabular}{|c|c|c|c|c|c|c|c|}
\hline \multirow[b]{2}{*}{$\begin{array}{l}\text { Clase social } \\
\text { del padre }\end{array}$} & \multicolumn{2}{|c|}{$\begin{array}{c}\text { Alumnos } \\
\text { presentados }\end{array}$} & \multicolumn{5}{|c|}{ Nota media expediente } \\
\hline & 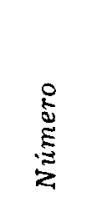 & $r$ & $\begin{array}{c}\mathscr{g} \\
\text { is } \\
0 \\
8 \\
8 \\
15 \\
0\end{array}$ & $\begin{array}{l}8 \\
0 \\
0 \\
8 \\
8 \\
0 \\
0\end{array}$ & 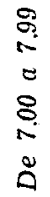 & $\begin{array}{l}8 \\
\infty \\
\infty \\
0 \\
8 \\
\infty \\
\infty \\
0\end{array}$ & $\begin{array}{l}\text { 으․ } \\
2 \\
0 .\end{array}$ \\
\hline $\begin{array}{llll}\text { Alta } \ldots & \ldots & \ldots & \ldots \\
\text { Media alta } \ldots & \ldots \\
\text { Media media } & \ldots \\
\text { Media baja } . & \ldots\end{array}$ & $\begin{array}{r}796 \\
11.832 \\
14.491 \\
19.367\end{array}$ & $\begin{array}{r}1 \\
16 \\
20 \\
26\end{array}$ & $\begin{array}{l}16 \\
16 \\
16 \\
14\end{array}$ & $\begin{array}{l}45 \\
44 \\
43 \\
45\end{array}$ & $\begin{array}{l}24 \\
27 \\
29 \\
29\end{array}$ & $\begin{array}{l}9 \\
8 \\
8 \\
9\end{array}$ & $\begin{array}{l}3 \\
3 \\
3 \\
3\end{array}$ \\
\hline 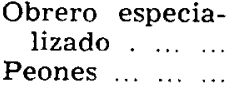 & $\begin{array}{r}21.690 \\
2.413\end{array}$ & $\begin{array}{r}29 \\
3\end{array}$ & $\begin{array}{l}14 \\
13\end{array}$ & $\begin{array}{l}45 \\
45\end{array}$ & $\begin{array}{l}30 \\
30\end{array}$ & $\begin{array}{l}8 \\
8\end{array}$ & $\begin{array}{l}3 \\
3\end{array}$ \\
\hline
\end{tabular}

Que las pruebas de selectividad no discriminan, en cuanto tales, gravemente a los alumnos de baja extracción social y que su supresión no cambiaría de forma notable el reclutamiento y compesición del alumnado universitario en contra de los estudiantes más modestos, se desprende también de los datos contenidos en los cuadros 11 y 12. El expediente académico de los alumnos procedentes de los estratos sociales más bajos es incluso un poco mejor, en términos generales, que el de los estudiantes de más elevada condición social. Un 41 por 100 de los estudiantes procedentes de familias de clase media-baja 
u obrera tienen una nota media de notable o sobresaliente, frente a un 38 por 100 y un 36 por 100 correspondientes a los de clase media alta o alta, respectivamente. De todos modos, hay que señalar que la tasa de aprobados es un poco más bajo entre los hijos de los obreros especializados y los peones que entre los hijos de las clases medias y altas, como puede verse en el cuadro 12 .

\section{CUADRO 12}

\section{Aprobados por clase social}

(Porcentaje de alumnos aprobados en las pruebas de acceso a la Universidad, 1979-1980, según clase social del padre)

\begin{tabular}{|c|c|c|}
\hline Clase social & de aprobados & $\begin{array}{l}\text { Diferencias } \\
\quad \operatorname{con} \bar{x}\end{array}$ \\
\hline $\begin{array}{lllllll}\ldots & \ldots & \ldots & \ldots & \ldots & \ldots & \ldots\end{array}$ & 77 & -2 \\
\hline $\begin{array}{llllllll}\text { Media } \text { alta } & \ldots & \ldots & \ldots & \ldots & \ldots & \ldots & \ldots\end{array}$ & 82 & +3 \\
\hline $\begin{array}{llllllll}\text { Media media } & \ldots & \ldots & \ldots & \ldots & \ldots & \ldots\end{array}$ & 81 & +2 \\
\hline $\begin{array}{llllllll}\text { Media baja } & \ldots & \ldots & \ldots & \ldots & \ldots & \ldots & \ldots\end{array}$ & 80 & +1 \\
\hline Obrero especializado $\ldots \ldots \ldots \ldots$ & 76 & -3 \\
\hline $\begin{array}{llllllllll}\text { Peón } & \ldots & \ldots & \ldots & \ldots & \ldots & \ldots & \ldots & \ldots & \ldots\end{array}$ & 76 & -3 \\
\hline
\end{tabular}

Cabe señalar que las diferencias de aprobados por clase social son realmente pequeñas. Los porcentajes más altos corresponden a las clases medias. Los más bajos a la clase alta y a las más bajas (obreros especializados y peones).

Está claro que el filtro es previo a la prueba de acceso, es decir, buena parte de los hijos de familias de obreros y peones no se inscriben para las pruebas de acceso. Aunque los que lo hacen no aprueban en la misma proporción que los hijos de familias más acomodadas, hay que señalar que la diferencia no es grande .

Mención especial mcrece el índice de fracasos académicos entre los hijos de familias de clase alta, aunque aquí no se dispone de datos explicativos del fenómeno.

: Somos conscientes de la provisionalidad de algunas de las afirmaciones conclusivas de este trabajo. Estamos pendientes de un análisis multivariable de los datos en los que se basa esta nota y sobre un material equivalente, relativo a las pruebas de selectividad para el curso 1980-1981, que ya está en trámite de proceso. En breve daremos cuenta de los resultados de estos análisis, revisando y completando lo que aquí se anticipa. En todo caso, la conclusión fundamental acerca de que la selectividad del alumnado se produce antes de las pruebas de acceso parece suficientemente probada. En segundo lugar, hay que decir que resulta problemático atribuir a variables concretas las diferencias de éxito en las pruebas de acceso. Si bien las cifras de aprobados resultan ligeramente desfavorables para los estudiantes de origen más modesto, no existen evidencias suficientes acerca de su carácter discriminatorio. 
En resumen, las pruebas de selectividad, en cuanto tales, no tienen un carácter marcadamente clasista, ni refuerzan en forma importante el elitismo real de la enseñanza universitaria en España. En todo caso, conforman simbólicamente una selección efectuada en los niveles anteriores del sistema educativo. Es en las enseñanzas primaria y media donde actúan con más fuerza los filtros selectivos que discriminan a los estudiantes de origen social modesto. De éstos, sólo los más aptos, o los que mejor han podido o han sabido adaptarse a las exigencias del sistema escolar, sobreviven a esa carrera de obstáculos y se enfrentan a las pruebas de acceso a la Universidad con probabilidades de éxito similares a las de sus compañeros de condición social más acomodada. Entre los factores que actúan de filtros selectivos cabe destacar, como se ha venido señalando a lo largo de estas páginas, la procedencia rural con lo que ella implica de lejanía de los mejores centros educativos, principalmente privados, de más bajo nivel de renta, de inferior nivel cultural medio de los padres e inferior estímulo académico de la familia y el entorno; dentro del hábitat urbano también operan esos mismos factores económicos, familiares y culturales del ambiente primario en que se desarrolla la vida del alumno. $\mathrm{Y}$ dado que el factor cultural-académico influye de forma importante, no se puede olvidar el carácter circular de este influjo, al menos desde el punto de vista de la marginación cultural creciente de los estratos más bajos frente a los medios y altos. Entre los más altos existen indicios en sentido contrario, pues su índice de fracasos en las pruebas de acceso a la Universidad es sorprendentemente alto, pero no son numéricamente significativos, al menos no hasta el punto de invertir la tendencia selectiva general del sistema educativo español, operada en los niveles previos a la Universidad. 


\section{CUADRO A}

Alumnos presentados y aprobados en las pruebas de acceso a la Universidad (1979-1980), por provincia

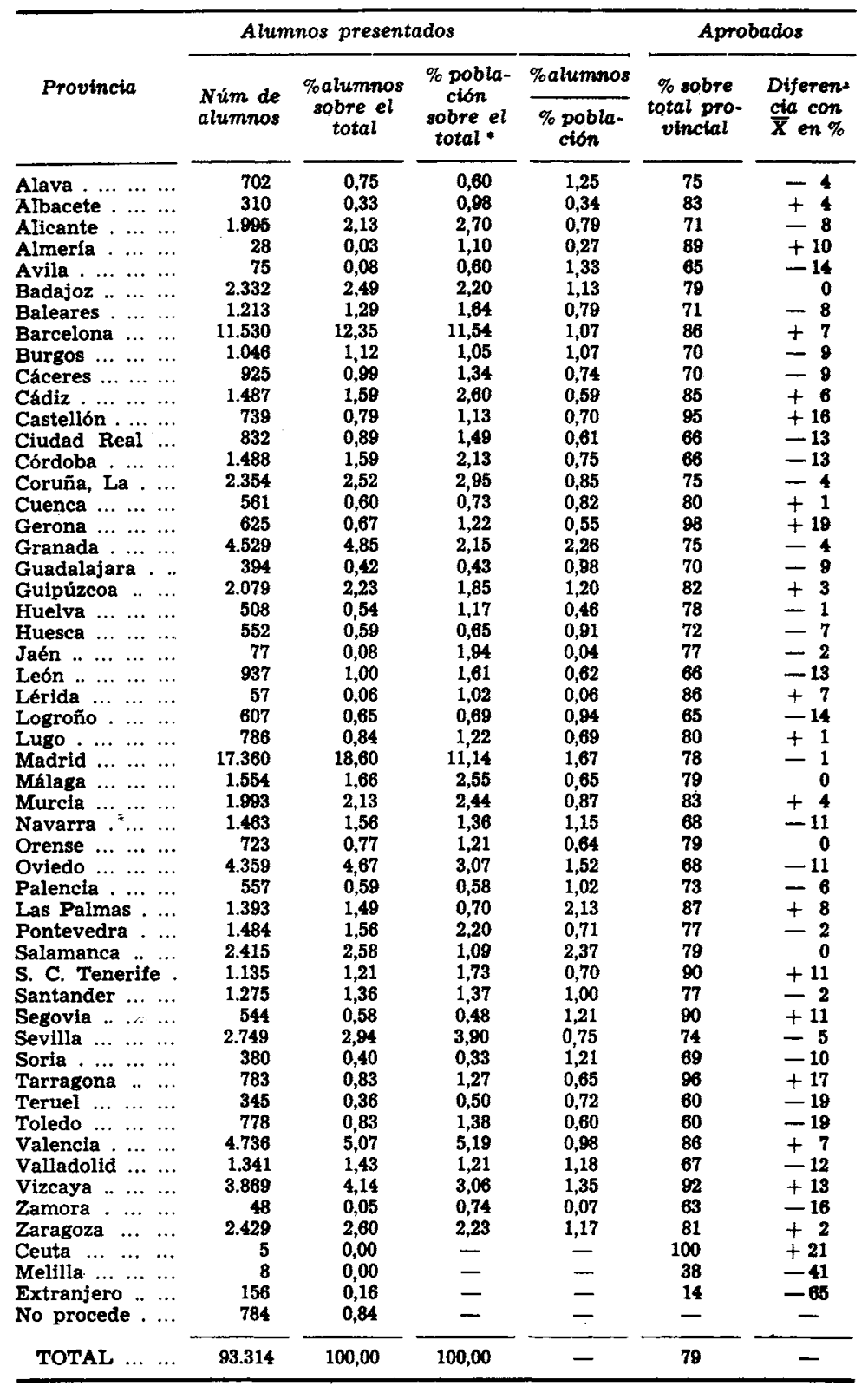

- I. N. E., Censo de Población, 1970. 


\section{CUADRO B}

\section{Alumnos presentados y aprobados por región}

(Distribución regional de alumnos presentados y aprobados en las pruebas de acceso a la Universidad, 1979-1980)

\begin{tabular}{|c|c|c|c|c|c|c|c|c|}
\hline \multirow[b]{2}{*}{ Región } & \multicolumn{4}{|c|}{ Alumnos presentados } & \multicolumn{4}{|c|}{ Aprobados } \\
\hline & 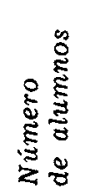 & 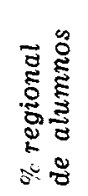 & 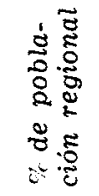 & 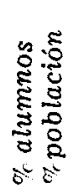 & $\begin{array}{l}\vec{\nabla} \\
\stackrel{0}{0} \\
\underbrace{2}\end{array}$ & 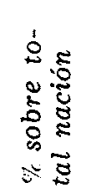 & 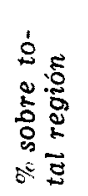 & 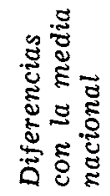 \\
\hline indalucía & 12.420 & 13,31 & 17,58 & 0,76 & 8.324 & 11,31 & 67,02 & $-11,78$ \\
\hline Aragón $\quad \ldots \ldots \ldots \ldots \ldots$ & 3.326 & 3,56 & 3,39 & 1,05 & 2.574 & 3,49 & 77,39 & $\ldots 1,41$ \\
\hline Asturias & 4.379 & 4,69 & 3,07 & 1,53 & 2.946 & 4,00 & 67,27 & $-11,53$ \\
\hline Baleares $\ldots \ldots \ldots \ldots c h$ & 1.213 & 1,30 & 1,64 & 0,80 & 863 & 1,17 & 71,14 & $-7,66$ \\
\hline $\begin{array}{lllll}\text { Barcelona } & \ldots & \ldots & \ldots & \ldots\end{array}$ & 11.530 & 12,36 & 11,57 & 1,07 & 9.905 & 13,46 & 85,90 & $+7,10$ \\
\hline Canarias & 2.528 & 2,71 & 3,44 & 0,79 & 2.277 & 3,09 & 90,07 & $+11,27$ \\
\hline Castilla la Nueva .. ... & 2.875 & 3,10 & 6,34 & 0,49 & 1.735 & 2,35 & 60,34 & $-18,46$ \\
\hline Castilla la Vieja .. ... & 5.825 & 6,24 & 5,02 & 1,25 & 4.230 & 5,75 & 72,61 & $-6,19$ \\
\hline Cataluña (resto) $\ldots \ldots$ & 1.465 & 1,57 & 3,51 & 0,45 & 1.411 & 1,91 & 96,31 & $+17,51$ \\
\hline $\begin{array}{llll}\text { Extremadura } & \ldots & \ldots & \ldots\end{array}$ & 3.262 & 3,49 & 3,37 & 1,04 & 2.491 & 3,38 & 76,36 & $-2,44$ \\
\hline Galicia ... ... & 5.227 & 5,71 & 7,60 & 0,76 & 4.095 & 5,56 & 76,87 & $-1,93$ \\
\hline León & 3.400 & 3,64 & 3,45 & 1,06 & 2.534 & 3,44 & 74,52 & $-4,28$ \\
\hline Madrid & 17.360 & 18,60 & 11,16 & 1,67 & 13.455 & 18,29 & 77,50 & $-1,30$ \\
\hline Murcia & 1.993 & 2,13 & 2,45 & 0,87 & 1.953 & 2,65 & 97,99 & $+19,19$ \\
\hline Navarra & 1.473 & 1,57 & 1,36 & 1,16 & 988 & 1,34 & 67,53 & $-11,27$ \\
\hline País Valenciano ... ... & 7.470 & 8,00 & 9,05 & 0,89 & 6.190 & 8,41 & 82,86 & $+4,06$ \\
\hline País Vasco $\ldots \ldots \ldots$ & 6.650 & 7,13 & $\mathbf{5 , 5 3}$ & 1,29 & 5.800 & 7,88 & 87,21 & $+8,41$ \\
\hline $\begin{array}{lllllll}\text { Resto } & \ldots & \ldots & \ldots & \ldots & \ldots & \ldots\end{array}$ & 828 & 0,88 & 0,47 & 1.88 & 1.780 & 2,42 & & \\
\hline TOTAL $\ldots \ldots \ldots$ & 93.314 & 100,00 & 100.00 & & 73.559 & 100,00 & 78,80 & \\
\hline
\end{tabular}

